\title{
Epistaxis: aspectos nuevos a considerar
}

\section{Epistaxis: new aspects to consider}

\author{
Nicolás Utrera Q. ${ }^{1}$, Hugo Romero $V^{1}$, Esperanza Salvo P. ${ }^{1}$, \\ Felipe Gauna P. ${ }^{1,2}$, Cristián Papuzinski A. ${ }^{1,2,3,4}$
}

\section{Resumen}

La epistaxis es una de las urgencias otorrinolaringológicas más frecuentes, y se estima que un $6 \%$ requerirá algún tipo de intervención. Según la localización del sitio de sangrado puede clasificarse en anterior, posterior o superior. A lo largo de la historia su manejo ha seguido ciertos ejes que se mantienen vigentes, como son la compresión nasal, posicionamiento de la cabeza, taponamiento nasal, entre otros. Tras la implementación de la evaluación endoscópica sistemática del sitio de sangrado, estudios han descrito un nuevo punto de sangrado denominado $S$-point, que debiese ser especialmente considerado en epistaxis severa. Además, durante los últimos años la evidencia ha revelado ciertos aspectos especiales y relevantes en torno a la evaluación y/o manejo de pacientes con epistaxis y condiciones asociadas, como el uso de terapia antitrombótica, telangiectasia hemorrágica hereditaria o con hipertensión arterial. El objetivo de esta revisión es resumir aspectos novedosos en la evaluación, estudio y manejo de la epistaxis, donde se incluirán el uso de ácido tranexámico y de nuevos dispositivos intranasales.

Palabras clave: Epistaxis, telangiectasia hemorrágica hereditaria, anticoagulantes, ácido tranexámico, hipertensión.

\begin{abstract}
Epistaxis is one of the most frequent otorhinolaryngological emergencies, and it is estimated that $6 \%$ require some type of intervention. Depending on the location of the bleeding site, it may be classified as anterior, posterior or superior. Throughout history, its treatment has remained the same, including nasal compression, head positioning, nasal packing, among others. After the implementation of the systematic endoscopic assessment of the bleeding site, studies have described a new point of bleeding called S-point, which should be especially considered in severe epistaxis. In addition, during the last years the evidence has revealed certain special and relevant aspects regarding the evaluation and/or management of patients with epistaxis and associated conditions, such as the use of antithrombotic therapy, hereditary hemorrhagic telangiectasia or arterial hypertension. The objective of this review is to summarize novel aspects in the evaluation, study and management of epistaxis, which include the use of tranexamic acid and new intranasal devices. Keywords: Epistaxis, hereditary hemorrhagic telangiectasia, anticoagulants, tranexamic acid, hypertension.
\end{abstract}

\author{
${ }^{1}$ Departamento de \\ Especialidades, Cátedra de \\ Otorrinolaringología, Escuela de \\ Medicina, Facultad de Medicina, \\ Universidad de Valparaíso. Viña \\ del Mar, Chile. \\ ${ }^{2}$ Servicio de Otorrinolaringología \\ del Hospital Carlos Van Buren, \\ Universidad de Valparaíso. \\ Valparaíso, Chile. \\ ${ }^{3}$ Centro Interdisciplinario de \\ Estudios en Salud (CIESAL), \\ Universidad de Valparaíso. \\ Valparaíso, Chile. \\ ${ }^{4}$ Departamento de Cirugía, \\ Facultat de Medicina i \\ Odontologia, Universitat de \\ València. València, España. \\ Los autores declaran no tener \\ conflictos de interés. \\ Recibido el 22 de septiembre de \\ 2020. Aceptado el 14 de marzo \\ de 2021. \\ Correspondencia: \\ Felipe Gauna P. \\ San Ignacio 725. \\ Valparaíso, Chile. \\ Email: felipe.gauna@postgrado. \\ uv.cl
}

\section{Introducción}

La epistaxis corresponde a un problema de salud frecuente, que se estima afecta hasta un $60 \%$ de las personas a lo largo de su vida ${ }^{1}$, pudiendo llegar a ser severa si el sangrado nasal es masivo y requerir un manejo urgente o inmediato ${ }^{2}$. El $70 \%$ será un episodio auto- limitado, pero hasta un $6 \%$ de los episodios requieren alguna intervención ${ }^{3}$, siendo ésta de tipo quirúrgica, un $3,5 \%$ de las veces ${ }^{2}$, y con una mortalidad a 30 días posterior al episodio de hasta un $34 \%{ }^{4}$.

En los servicios de urgencia, la epistaxis representa un $0,5 \%$ de todos los motivos de consulta y hasta un tercio de las consultas otorri- 
nolaringológicas ${ }^{5}$, situándose como una de las emergencias más frecuentes de la especialidad ${ }^{6}$. Sumado a esta importancia clínica, los costos de atención en pacientes que se presentan con epistaxis son muy variados, aumentando significativamente cuando requieren intervenciones como taponamiento nasal, llegando a ser muy altos si se requiere hospitalización o cirugía $a^{5,7}$. Epidemiológicamente, se describen dos grupos etarios predominantes, niños entre 2 a 10 años y adultos entre 45 a 65 años ${ }^{8}$. Respecto al sexo, algunas fuentes describen una frecuencia más alta en el sexo masculino ${ }^{9}$, mientras que otras no refieren una clara diferencia ${ }^{5}$.

Existen distintas formas de clasificar a la epistaxis. En cuanto a su origen, pueden dividirse en epistaxis anteriores (90\%-95\%), posteriores $(5 \%-7 \%)$ y superiores $(1 \%)^{10}$. Otra forma es agruparlas en primarias o idiopáticas $(85 \%)$ y secundarias (a trauma, uso de anticoagulante, cirugías, entre otras causas reconocibles) ${ }^{6,8}$. Así, las etiologías son múltiples y se pueden agrupar en distintos

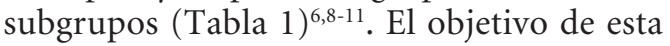
revisión es resumir algunos aspectos novedosos en la evaluación, estudio y manejo de la epistaxis.

\section{Manejo general en epistaxis}

El 2020, la American Academy of Otolaryngology-Head and Neck Surgery publicó una guía de práctica clínica para el manejo de la epistaxis dirigida a médicos generales y especialistas, acuñando un algoritmo de manejo basado en la mejor evidencia disponible (Figura 1).

Se dice que el 65\%-75\% de las epistaxis que requieren algún tipo de intervención pueden ser resueltas con medidas generales por un médico de atención primaria o de urgencia ${ }^{12}$. El manejo inicial debe incluir la compresión bidigital en el tercio inferior de la nariz por 5 minutos o más, previo retiro del coágulo nasal, con la cabeza en flexión anterior para evitar la deglución de sangre. Además, se señala que el uso de vasoconstrictores tópicos puede ser de utilidad $^{5,12}$. Se debe proceder a taponamiento nasal anterior o cauterización en aquellos pacientes con persistencia del sangrado pese a la compresión digital o en aquellos donde esta maniobra difícilmente detendrá el sangrado ${ }^{5}$. Una vez que el sangrado haya sido controlado, se debe identificar el sitio del sangrado mediante una rinoscopía anterior ${ }^{5}$. En algunos casos, si el sangrado no se logra controlar, se pueden realizar medidas más invasivas como taponamiento posterior, embolización o manejo quirúrgico ${ }^{13}$.

Además, de manera transversal a cualquier tipo de manejo, deben resguardarse las precauciones mínimas de contaminación (mascarilla, guantes, antiparras), asegurar la vía aérea, vigilar el estado hemodinámico del paciente y obtener una buena historia clínica buscando factores de riesgo para epistaxis (cirugía nasal o sinusal previa, trauma nasal o facial, uso de drogas o medicamentos intranasales, historia personal o familiar de desórdenes de hemostasia, entre otros) $)^{5,12}$.

Tabla 1. Etiologías frecuentes de epistaxis

\begin{tabular}{ll}
\hline Subgrupo & Causas \\
Locales & $\begin{array}{l}\text { Traumáticas (manipulación digital, contusión, fractura nasal, cuerpo extraño, iatrogénica), en- } \\
\text { fermedades inflamatorias (rinitis alérgica), infecciones, estructurales (sequedad de la mucosa, } \\
\text { perforación septal), neoplásicas (angiofibroma nasofaríngeo juvenil, tumores de la cavidad nasal y } \\
\text { senos paranasales) }\end{array}$ \\
Sistémicas & $\begin{array}{l}\text { Vasculopatías, telangectasia hemorrágica hereditaria, hematológicas (trombocitopenia, hemofilia } \\
\text { A, hemofilia B, enfermedad de Von Willebrand), enfermedades cardiovasculares, cirrosis hepática, }\end{array}$ \\
hmbientalen, enfermedad renal, déficit de vitamina K
\end{tabular}




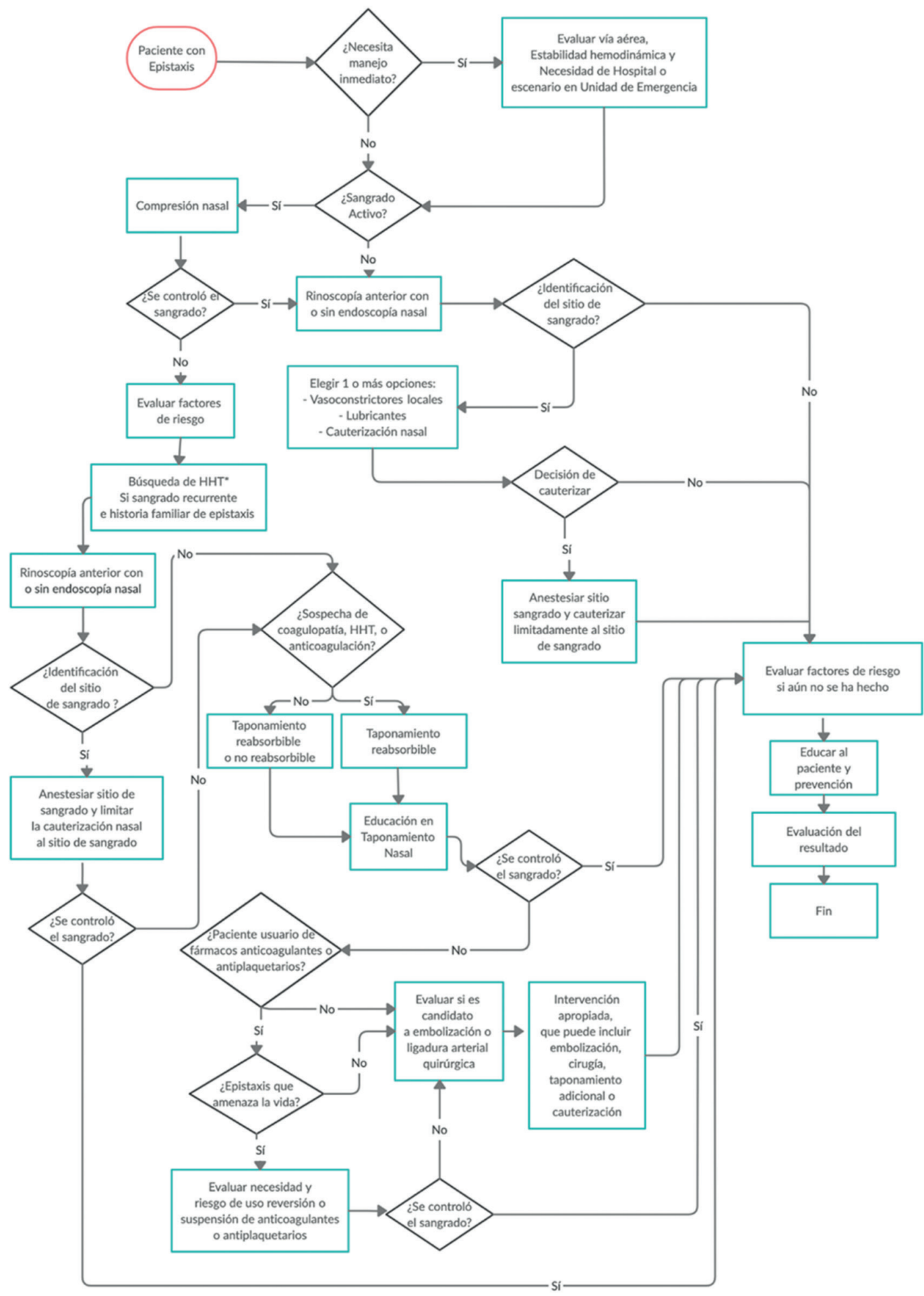

Figura 1. Algoritmo de evaluación y manejo de epistaxis. Traducido y adaptado de Tunkel DE, Anne S, Payne SC, et al. Clinical Practice Guideline: Nosebleed (Epistaxis). Otolaryngol Head Neck Surg 2020;162(1_suppl): S32. * HHT: Telangiectasia hemorrágica hereditaria. 


\section{"S-point": un sitio a considerar}

Como fue mencionado, las epistaxis pueden ser anteriores o posteriores según la localización del punto de sangrado ${ }^{14}$, divididas anatómicamente por la apertura piriforme ${ }^{6}$ y se ha propuesto que un $1 \%$ de las epistaxis serían superiores ${ }^{10}$. Sin embargo, algunos estudios han descrito que los sangrados superiores serían subdiagnosticados, reportando que hasta un $36 \%$ de las epistaxis posteriores se originan a nivel del septum superior ${ }^{2,15}$.

El año 2017, un grupo brasileño, liderado por el Dr. Aldo Stamm, publicó un artículo haciendo referencia a sangrados provenientes de la rama septal de la arteria etmoidal anterior, la cual se ubica en el septum nasal superior, en la proyección de la axila del cornete medio; este punto fue denominado "S point" en honor al autor mencionado ${ }^{16}$. Poco tiempo después, un grupo italiano describió el mismo sitio con una serie de $\operatorname{casos}^{17}$. Este pedículo vascular arterial, sería origen de epistaxis severa ${ }^{16}$ y refractaria en un alto porcentaje de los $\operatorname{casos}^{17}$. Una serie de casos reciente con 51 pacientes con epistaxis severa, los cuales se sometieron a evaluación endoscópica sistemática del sitio de sangrado, se reportó que la mitad de los sangrados eran de origen superior, siendo el lugar más frecuente encontrado el "S point", correspondiendo a un $28 \%$ del total ${ }^{2}$. En esta misma serie de casos, se sugiere realizar una búsqueda activa y sistemática del sitio de sangrado, con énfasis en el "S point" ya que puede ser de difícil acceso y en general poco explorado ${ }^{16}$. Se sugiere la exploración sin uso de descongestionantes y sin hipotensión, ya que podrían esconder el punto sangrante ${ }^{2,16}$ y su posterior cauterización local ${ }^{16}$, siendo una alternativa segura y efectiva en 3 series de casos publicados ${ }^{2,16,17}$.

Tomando en cuenta lo anterior, ilustramos esto con un caso de una mujer de 75 años, usuaria de ácido acetilsalicílico que fue evaluada ambulatoriamente en nuestro centro por epistaxis recurrente, donde se identificó el sangrado proveniente de la rama septal de la arteria etmoidal anterior y se cauterizó bajo anestesia local con nitrato de plata, logrando control definitivo del sangrado (Figura 2).

Figura 2. A: Identificación del sitio de sangrado, detrás del tubérculo septal. B: Se observa sangrado de la rama septal de la AEA ("S point") en la proyección de la axila del cornete medio (flecha blanca). C: Se observa el sangrado hacia la nasofaringe, lo que podría confundir con sangrados de origen posterior. D: Cauterización endoscópica con nitrato de plata al sitio sangrante. * CM: cornete medio; TS: tubérculo septal; $\mathrm{Cl}$ : cornete inferior; NF: nasofaringe; NP: nitrato de plata; AEA: arteria etmoidal anterior.

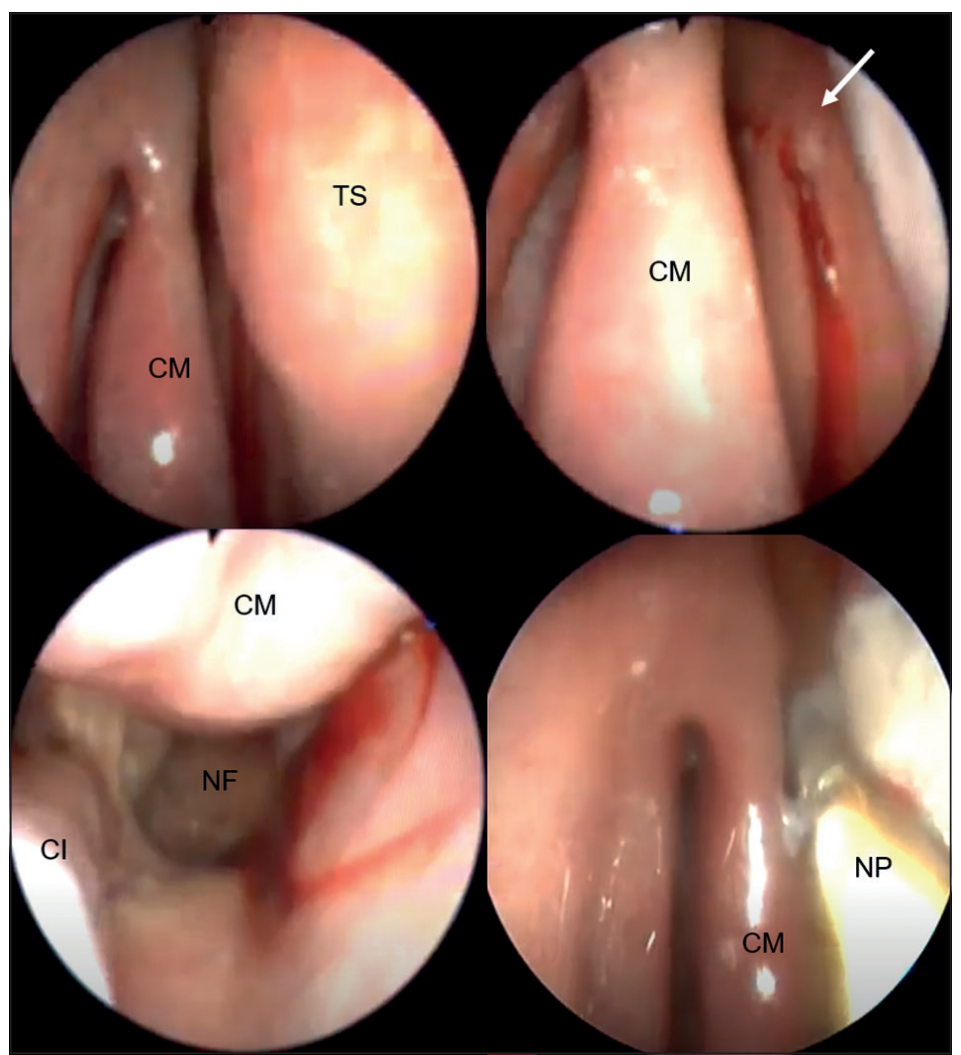




\section{Ácido tranexámico}

Se ha propuesto el uso de ácido tranexámico (ATX) para el manejo de la epistaxis aguda, tanto por vía oral ${ }^{18}$, como por vía tópica ${ }^{19}$. En estudios que evaluaron la efectividad del uso de ATX por vía oral, el esquema más utilizado fue de 1 gramo cada 8 horas por 10 días $^{18}$, mientras que para la vía tópica existen diferentes esquemas propuestos. Zahed y cols. realizaron dos ensayos clínicos aleatorizados donde se colocó algodón empapado con ATX en su forma inyectable ( $500 \mathrm{mg}$ en $5 \mathrm{~mL}$ ), para luego retirarlo una vez que el sangrado se haya detenido ${ }^{19,20}$. Una revisión Cochrane comparó el uso de ATX con placebo o manejo usual con otros agentes hemostáticos. Esta síntesis concluye que cuando se compara con placebo o manejo usual, el ATX reduce el riesgo de resangrado a los 10 días (RR 0,71 (IC 95\% 0,56 a 0,90; $\mathrm{I} 2=0 \%)$ ), y cuando se compararon ambas vías de administración, sólo la vía oral mostró efectividad (RR 0,73 IC 95\% 0,55 a 0,96). No se encontraron diferencias en control agudo de la epistaxis (dentro de los primeros 30 minutos) (RR 0,79 IC 95\% 0,56 $\mathrm{a}^{1,11}$ ). Por otro lado, cuando se comparó el ATX con otros agentes hemostáticos como epinefrina y lidocaína, el ATX por vía tópica demostró ser más efectivo en el control agudo del sangrado (detención del sangrado dentro de los primeros $10 \mathrm{mi}$ nutos) (RR 2,35 IC 95\% 1,90 a 2,92;I2 = 0\%). En ninguno de los estudios se reportaron eventos adversos mayores ${ }^{21}$. Una segunda revisión sistemática evaluó el efecto del ATX por vía tópica y concluyó que si bien no hubo diferencias estadísticamente significativas en control agudo del sangrado (detención del sangrado dentro de los primeros 30 minutos), sí hubo mayores egresos hospitalarios a las dos primeras horas con respecto al grupo control, y un menor número de episodios de resangrado a las 24 horas y 1 semana con respecto al grupo control $^{22}$.

\section{Telangiectasia hemorrágica hereditaria: una causa a considerar}

El síndrome de telangiectasia hemorrágica hereditaria $(\mathrm{THH})$ o enfermedad de Rendu-Osler-Weber, se caracteriza por una historia familiar epistaxis a repetición, telan- giectasias y malformaciones arteriovenosas mucocutáneas y viscerales ${ }^{23}$. Esta enfermedad es considerada un tópico aparte en el manejo de epistaxis dada su naturaleza. Durante los últimos 25 años, tras el descubrimiento de las mutaciones asociadas a la enfermedad, se han realizado numerosos avances en las alternativas terapéuticas ${ }^{24}$; pero en la actualidad no existe consenso, ya que existen pocos estudios prospectivos aleatorizados y el tratamiento depende de las características particulares del paciente y los protocolos de cada centro ${ }^{25}$.

Aunque aún no existe evidencia suficiente para recomendar su uso, existen múltiples drogas en estudio que han mostrado efectos prometedores, evidenciando mejoras tanto en puntajes de calidad de vida, como en criterios objetivos (número de sangrados, necesidad de transfusiones, manejo de anemia, etc. $)^{24,26}$, por lo que se incluirá en la revisión algunas novedades en el manejo de esta patología.

\section{Manejo de epistaxis en THH}

\section{Manejo de epistaxis aguda en $\mathrm{THH}$}

En estos pacientes la epistaxis aguda siempre debe considerarse como un episodio potencialmente grave, ya que, al experimentar sangrados frecuentes, la mayoría de las veces saben controlarlos de forma autónoma y solo consultan en episodios refractarios o de mayor cuantía $^{27}$. De todas formas, se debe realizar un abordaje escalonado, al igual que en otros pacientes, partiendo por la compresión nasal y progresando a medidas más invasivas en caso de no lograr el control ${ }^{5,27}$. Se debe evitar el uso de taponamientos no reabsorbibles por el riesgo de resangrado al retirarlos ${ }^{5,28}$, por ejemplo, SurgiCel ${ }^{\circledR} \mathrm{O}$ GelitaCel ${ }^{\circledR 27}$. En caso de falla con el taponamiento se podría intentar embolización de la arteria facial y maxilar interna ${ }^{27}$.

\section{Manejo médico en THH: nuevas alternativas}

Se han desarrollado distintas estrategias para buscar nuevos fármacos que ayuden a controlar los sangrados en estos pacientes: 1) Estabilizar la red de fibrina con antifibrinolíticos (ácido tranexámico y ácido aminocaproico); 2) Estimular la transcripción de genes probablemente involucrados (moduladores del receptor específico de estrógeno (SERMs), tacrolimus, n-acetil cisteína); 3) Antiangio- 
génesis (bevacizumab, propranolol, timolol, talidomida, pazopanib, etamsilato). Existen además otras drogas como itraconazol, doxiciclina y vitamina $\mathrm{D}$ que se encuentran en estudio ${ }^{26}$ :

- Ácido tranexámico: de forma sistémica en pacientes con bajo riesgo de trombosis ha demostrado efectividad, elevando niveles de hemoglobina y reduciendo necesidad de transfusiones. De forma tópica, no ha logrado demostrar utilidad ${ }^{26}$. Tiene potencial de reducir frecuencia, intensidad y duración de sangrados ${ }^{25}$.

- Moduladores del receptor específico de estrógeno (SERMs): han demostrado efectividad en el manejo de sangrados gastrointestinales y epistaxis, aumentando niveles de hemoglobina y reduciendo necesidad de transfusiones ${ }^{26,29}$.

- Bevacizumab (anti-VEGF): en pacientes con epistaxis severa, sin opción de otros tratamientos, ha demostrado reducir los episodios. Se debe tener en consideración el potencial de serios efectos adversos y que el primer estudio clínico aleatorizado en fase III se encuentra en realización ${ }^{24,26,30}$.

- Talidomida: en dosis bajas (50 mg al día) y con incremento cada 4 semanas si no hay respuesta, ha demostrado utilidad en reducir frecuencia, intensidad y duración de sangrados nasales, así como incrementar niveles de hemoglobina. Debe evaluarse el riesgo versus beneficio por sus efectos $\operatorname{adversos}^{29}$.

- Timolol y propranolol tópico: han demostrado disminuir la frecuencia y severidad de la epistaxis ${ }^{26}$.

\section{Tratamientos quirúrgicos en THH}

Dentro de los tratamientos quirúrgicos, se debe evitar la cauterización con nitrato de plata y electrocauterización monopolar porque se ha asociado a mayor riesgo de perforación septal y empeoramiento del sangrado ${ }^{25}$. En pacientes con epistaxis moderada a severa se puede considerar electrocauterización bipolar, coagulación electroquirúrgica con plasma (coblation) y fotocoagulación con láser ${ }^{25}$. Una revisión sistemática reciente, demostró alta tasa de disminución de la frecuencia y severidad de epistaxis al utilizar láser de argón y Nd:YAG, con porcentajes menores para el láser de diodo ${ }^{31}$ En epistaxis severa el Nd:YAG sería superior a $\operatorname{argón}^{31}$. Para los láser KTP y PDL aún existe poca evidencia para el análisis cuantitativo $^{31}$.

Existen otras opciones más agresivas, como la septodermoplastía, que corresponde a la remoción de la capa mucosa superficial del septum y que se puede extender al piso de la fosa nasal, el cornete inferior y pared lateral nasal según la extensión de la enfermedad. Posteriormente se coloca un injerto de piel parcial para cubrir el defecto generado ${ }^{25}$. Este procedimiento ha demostrado ser efectivo en el control de la epistaxis al menos a 2 años, pero está asociado a costras, mal olor y necesidad de cuidados nasales diarios ${ }^{25}$, por lo que no está recomendada en la última guía francesa de manejo de epistaxis en $\mathrm{THH}^{27}$. Otra opción es el cierre nasal (procedimiento de Young), que consiste en el cierre del vestíbulo nasal mediante el levantamiento de colgajos mucocutáneos. Este procedimiento ha demostrado efectividad en control de epistaxis, pero está asociado a pérdida permanente del olfato y respiración oral de por vida ${ }^{25,27,32}$. Estos últimos 2 procedimientos deben ser reservados para pacientes con epistaxis refractaria a otros manejos por sus consecuencias permanentes e importantes efectos en la calidad de vida.

\section{Epistaxis y tratamiento antitrombótico}

El uso de tratamiento antitrombótico (TAT), ya sea como antiagregante plaquetario o como anticoagulante (AC), se ha asociado a mayor riesgo de epistaxis y de episodios recurrentes, mientras que no se ha asociado a una mayor severidad de estos ${ }^{33-35}$. La guía clínica de la Société Française d'Oto-Rhino-Laryngologie et de Chirurgie de la Face et du Cou, para el manejo de epistaxis en pacientes usuarios de TAT, recomienda que se debe estimar el riesgo de trombosis y el riesgo de sobredosis en cada paciente usuario de TAT que consulta con epistaxis. Para aquellos pacientes usuarios de AC con antagonistas de la vitamina $\mathrm{K}$ con epistaxis aguda se recomienda monitorización de la terapia con International Normalized Ratio (INR) para descartar sobredosis. Clásicamente, la compresión digital inicial se realiza por 5 minutos o más, pero en estos 
pacientes, el enfrentamiento inicial debería ser compresión digital por un mínimo de 10 minutos $y$, en caso de que persista, proceder a medidas más invasivas. Aquellos casos severos deberían hospitalizarse, incluyendo la participación de un hematólogo o cardiólogo para su evaluación. Si el taponamiento es efectivo y/o el paciente tiene un alto riesgo trombótico, el TAT debe ser continuado. En los casos donde exista persistencia o recurrencia del sangrado, se recomienda considerar modificaciones de la terapia, suspensión de la terapia, administración de vitamina $\mathrm{K}$ o uso de antídotos en anticoagulantes orales de acción directa en conjunto con hematología y/o cardiología ${ }^{36}$. La guía clínica de la American Academy of Otolaryngology-Head and Neck Surgery sobre el manejo de la epistaxis sugiere el uso de material reabsorbible para el taponamiento nasal en pacientes con coagulopatías o usuarios de $\mathrm{TAT}^{5}$.

\section{Dispositivos de taponamiento nasal}

En pacientes cuya epistaxis no cede luego de compresión nasal, se continúa con taponamiento nasal para controlar el sangrado, utilizando diversos materiales no absorbibles y reabsorbibles ${ }^{5}$. Pese a existir una gran variedad de materiales, existen escasos análisis comparativos de efectividad ${ }^{5,37}$.

\section{FloSeal $^{\circledR}$}

El más estudiado es el FloSeal, una matriz hemostática gelatinosa reabsorbible impregnada en trombina ${ }^{5,37}$. Puede ser utilizado en el servicio de urgencias por médicos no especialistas entrenados ${ }^{5,37}$. En epistaxis anterior, el FloSeal demostró utilidad en controlar el sangrado y satisfacción del paciente, sin embargo, se evaluaron con parámetros subjetivos y en un reducido número de pacientes ${ }^{38}$. Posteriormente, otros estudios lo han comparado con taponamiento nasal convencional, concluyendo que no presenta diferencias estadísticamente significativas en control de sangrado ${ }^{39,40}$.

En cuanto a su costo-efectividad, un estudio que comparó el uso de FloSeal con taponamiento nasal convencional para el manejo de la epistaxis anterior, señala que, si bien el FloSeal es más costoso que el taponamiento nasal, concluye que es una alternativa costo-efectiva especialmente en aquellos centros dispuestos a pagar un mayor precio unitario ${ }^{41}$.

\section{Rapid Rhino ${ }^{\circledR}$}

El RapidRhino es un balón inflable con cobertura hidrocoloide principalmente utilizado en epistaxis posteriores ${ }^{42}$. Un estudio con 52 pacientes lo comparó con MeroCel ${ }^{\circledR}$ en epistaxis anterior, evidenciando propiedades hemostáticas similares, pero con mejor tolerancia a su inserción y retiro ${ }^{43}$.

\section{Hipertensión arterial: una patología a considerar}

La asociación entre epistaxis e hipertensión siempre ha sido controvertida ${ }^{44}$. En estudios que han evaluado esta asociación, se ha encontrado que esta asociación es más frecuente en hombres $(1,6: 1)$, ocurre el sangrado principalmente durante meses de invierno (54\%) y en pacientes que al consultar presentan mayor presión sistólica media ${ }^{45}$.

En distintas publicaciones se ha evidenciado que efectivamente existe una asociación entre ambas entidades, pero sin posibilidad de establecer causalidad, debido a la alta heterogeneidad y baja calidad de los estudios ${ }^{46,47}$. La guía americana de práctica clínica de epistaxis publicó un documento sobre la relación entre epistaxis e hipertensión, reafirmando la existencia de la asociación entre ambas, destacando que es posible que esta asociación se deba al daño vascular secundario a la elevación crónica de la presión arterial y que durante el manejo agudo de un paciente con epistaxis e hipertensión, disminuir bruscamente la presión arterial puede llevar a daño orgánico por hipoperfusión, debiendo esta conducta evaluarse caso a caso, pues no existe evidencia que estadísticamente apruebe o refute este manejo ${ }^{48}$.

\section{Discusión}

Las medidas generales logran controlar hasta un $75 \%$ de los episodios de epistaxis que requieren atención médica, por lo que siguen estando vigentes en la actualidad ${ }^{12}$. La educación de la población, además del entrenamiento basado en competencias dentro de la formación médica, podría reducir el número de consultas en la urgencia y de pro- 
cedimientos u hospitalizaciones innecesarias, lo que lograría reducir los costos asociados a esta patología.

Consideramos que el enfrentamiento clásico que divide epistaxis en anteriores y posteriores es poco práctico, ya que no se enfoca en la gravedad del cuadro ni en la capacidad de controlar el sangrado con un abordaje escalonado. Al enfrentar una epistaxis se debe incluir la valoración de la vía aérea y la estabilidad de la hemodinamia, para posteriormente intentar ubicar el sitio exacto de sangrado en los casos que no se logre controlar con medidas generales. La evidencia reciente demuestra que un porcentaje considerable de sangrados provienen de la porción superior de la fosa nasal ${ }^{16,17}$, sitio que no siempre es explorado, lo que ha generado un cambio de paradigma, sobre todo en el manejo quirúrgico ${ }^{2,15,16}$.

Con respecto al uso de nuevos dispositivos, pueden constituir una herramienta importante en el manejo de la epistaxis dada su fácil aplicación, especialmente por médicos con poca experiencia ${ }^{37}$. Sin embargo, llama la atención la falta de estudios comparativos de efectividad entre estos ${ }^{5,37}$, siendo su utilización sujeta a criterio del médico, disponibilidad de cada centro y costos del dispositivo.

Existe evidencia que el uso de ATX por vía tópica y por vía oral podría ser útil en el control agudo de la epistaxis, estando la forma inyectable de este fármaco, la que se puede usar tópicamente, disponible en la mayoría de los servicios de urgencia ${ }^{21}$.

El manejo adecuado de las epistaxis en pacientes con telangiectasia hemorrágica hereditaria significa un desafío en el manejo para el otorrinolaringólogo y existe mucha investigación nueva en este campo, con drogas prometedoras que podrían mejorar la calidad de vida de estos pacientes ${ }^{25,26}$.

En pacientes usuarios de tratamiento antitrombótico con epistaxis, se debe estimar el riesgo de trombosis y de sobredosis. En la mayoría de ellos, su manejo inicial no difiere del de otros pacientes, pero en aquellos con persistencia o recurrencia del sangrado, se debe considerar la participación temprana de un cardiólogo o hematólogo para la eventual suspensión o modificación de la terapia ${ }^{36}$.

Finalmente cabe destacar que la asociación causal entre hipertensión arterial y epistaxis no ha podido ser respaldada en la evidencia ${ }^{46}$, y actualmente el foco entre estas entidades va dirigido a identificar a la epistaxis como marcador de enfermedad ateroesclerótica en pacientes hipertensos ${ }^{49,50}$.

\section{Conclusión}

La epistaxis es uno de los motivos de consulta y urgencia otorrinolaringológica más frecuentes, sin embargo, en los últimos años, nuevas publicaciones han llevado a un cambio en el enfrentamiento y los paradigmas clásicamente aceptados en torno a ella. Si bien algunos de estos temas cuentan con bastante evidencia como el uso de ácido tranexámico o el manejo en pacientes usuarios de tratamiento antitrombótico, aún hacen falta desarrollo en otros temas, como estudios comparativos y de costo efectividad en dispositivos intranasales o el rol del manejo de la hipertensión arterial en pacientes con epistaxis, además de recopilar más experiencias en torno al "S-point" para el manejo dirigido del control del sangrado.

\section{Bibliografía}

1. Retuert R, Fuentealba D, Bretón A, Ricci L, Nazar $\mathrm{R}$, Naser A. Manejo de epistaxis posterior en el Hospital Clínico de la Universidad de Chile entre los años 2013 y 2016. Rev Otorrinolaringol Cir Cabeza Cuello. 2017;77:389-394. doi: 10.4067/s071848162017000400389.

2. Loures CN, Castro TC, Luz Matsumoto GR, et al. Systematic endoscopic assessment of bleeding sites in severe epistaxis: the role of the S-point and the superior epistaxis. Rhinology. 2020;10.4193/ Rhin19.466. doi: 10.4193/Rhin19.466.

3. Villwock JA, Jones K. Recent trends in epistaxis management in the United States: 2008-2010. JAMA Otolaryngol Head Neck Surg. 2013;139(12):1279-1284. doi: 10.1001/jamaoto.2013.5220.

4. National ENT Trainee Research Network. Epistaxis 2016: national audit of management. J Laryngol Otol. 2017;131(12):1131-1141. doi: 10.1017/ S002221511700202X.

5. Tunkel DE, Anne S, Payne SC, et al. Clinical Practice Guideline: Nosebleed (Epistaxis). Otolaryngol Head Neck Surg. 2020;162(1_suppl):S1-S38. doi: 10.1177/0194599819890327F.

6. Passali D, Damiani V, Passali FM, Tosca MA, Motta G, Ciprandi G. An International Survey on the 
pragmatic management of epistaxis. Acta Biomed. 2020;91(1-S):5-10. doi: 10.23750/abm.v91i1-S.9241.

7. Cohen O, Shoffel-Havakuk H, Warman M, et al. Early and Late Recurrent Epistaxis Admissions: Patterns of Incidence and Risk Factors. Otolaryngol Head Neck Surg. 2017;157(3):424-431. doi: 10.1177/0194599817705619.

8. Wong AS, Anat DS. Epistaxis: A guide to assessment and management. J Fam Pract. 2018;67(12):E13-E20.

9. Kuo CL. Updates on the Management of Epistaxis. Clinical Medicine and Therapeutics. 2019;1(1):5. doi: 10.24983/scitemed.cmt.2019.00106.

10. García-Cabo P, Fernández-Vañes L, Pedregal D, et al. Manejo de las epistaxis graves y/o refractarias. Acta Otorrinolaringol Esp. 2019;70(4):185-191. doi: 10.1016/j.otorri.2018.02.004.

11. Lin G, Bleier B. Surgical Management of Severe Epistaxis. Otolaryngol Clin North Am. 2016;49(3):627637. doi: 10.1016/j.otc.2016.01.003.

12. Beck R, Sorge M, Schneider A, Dietz A. Current Approaches to Epistaxis Treatment in Primary and Secondary Care. Dtsch Arztebl Int. 2018;115(1-02):1222. doi: 10.3238/arztebl.2018.0012.

13. Shargorodsky J, Bleier BS, Holbrook EH, et al. Outcomes analysis in epistaxis management: development of a therapeutic algorithm. Otolaryngol Head Neck Surg. 2013;149(3):390-398. doi: 10.1177/0194599813492949.

14. Schlosser RJ. Clinical practice. Epistaxis. $N$ Engl J Med. 2009;360(8):784-789. doi: 10.1056/ NEJMcp0807078.

15. Chiu TW, McGarry GW. Prospective clinical study of bleeding sites in idiopathic adult posterior epistaxis. Otolaryngol Head Neck Surg. 2007;137(3):390-393. doi: 10.1016/j.otohns.2006.10.035.

16. Kosugi EM, Balsalobre L, Mangussi-Gomes J, et al. Breaking paradigms in severe epistaxis: the importance of looking for the S-point. Braz J Otorhinolaryngol. 2018;84(3):290-297. doi: 10.1016/j. bjorl.2017.12.007.

17. Turri-Zanoni M, Arosio AD, Stamm AC, et al. Septal branches of the anterior ethmoidal artery: anatomical considerations and clinical implications in the management of refractory epistaxis. Eur Arch Otorhinolaryngol. 2018;275(6):1449-1456. doi: 10.1007/s00405-018-4964-x.

18. White A, O'Reilly BF. Oral tranexamic acid in the management of epistaxis. Clin Otolaryngol Allied Sci. 1988;13(1):11-16. doi: 10.1111/j.1365-2273.1988. tb00275.x.

19. Zahed R, Moharamzadeh P, Alizadeharasi S, Ghasemi A, Saeedi M. A new and rapid method for epistaxis treatment using injectable form of tranexamic acid topically: a randomized controlled trial. Am J Emerg Med. 2013;31(9):1389-1392. doi: 10.1016/j. ajem.2013.06.043.

20. Zahed R, Mousavi Jazayeri MH, Naderi A,
Naderpour Z, Saeedi M. Topical Tranexamic Acid Compared With Anterior Nasal Packing for Treatment of Epistaxis in Patients Taking Antiplatelet Drugs: Randomized Controlled Trial. Acad Emerg Med. 2018;25(3):261-266. doi: 10.1111/ acem.13345.

21. Joseph J, Martinez-Devesa P, Bellorini J, Burton MJ. Tranexamic acid for patients with nasal haemorrhage (epistaxis). Cochrane Database Syst Rev. 2018;12(12):CD004328. doi: 10.1002/14651858. CD004328.pub3.

22. Gottlieb M, DeMott JM, Peksa GD. Topical Tranexamic Acid for the Treatment of Acute Epistaxis: A Systematic Review and Metaanalysis. Ann Pharmacother. 2019;53(6):652-657. doi: $10.1177 / 1060028018820625$.

23. Shovlin CL, Guttmacher AE, Buscarini E, et al. Diagnostic criteria for hereditary hemorrhagic telangiectasia (Rendu-Osler-Weber syndrome). Am J Med Genet. 2000; 91(1):66-67.doi: 10.1002/(sici)10968628(20000306)91:1<66::aid-ajmg12>3.0.co;2-p.

24. Robert F, Desroches-Castan A, Bailly S, DupuisGirod S, Feige JJ. Future treatments for hereditary hemorrhagic telangiectasia. Orphanet J Rare Dis. 2020;15(1):4. doi: 10.1186/s13023-019-1281-4.

25. Sautter NB, Smith TL. Treatment of Hereditary Hemorrhagic Telangiectasia-Related Epistaxis. Otolaryngol Clin North Am. 2016;49(3):639-654. doi: 10.1016/j.otc.2016.02.010.

26. Albiñana V, Cuesta AM, Rojas-P I, et al. Review of Pharmacological Strategies with Repurposed Drugs for Hereditary Hemorrhagic Telangiectasia Related Bleeding. J Clin Med. 2020;9(6):1766. doi: 10.3390/ jcm9061766.

27. Robard L, Michel J, Prulière Escabasse V, et al. Guidelines of the French Society of Otorhinolaryngology (SFORL) (short version). Specific treatment of epistaxis in Rendu-Osler-Weber disease. Eur Ann Otorhinolaryngol Head Neck Dis. 2017;134(1):37-41. doi: 10.1016/j.anorl.2016.09.010.

28. Riera-Mestre A, Ribas J, Castellote J. Medical management of haemorrhagic hereditary telangiectasia in adult patients. Tratamiento de la telangiectasia hemorrágica hereditaria en el paciente adulto. Med Clin (Barc). 2019;152(7):274-280. doi: 10.1016/j.medcli.2018.09.015.

29. Halderman AA, Ryan MW, Clark C, et al. Medical treatment of epistaxis in hereditary hemorrhagic telangiectasia: an evidence-based review. Int Forum Allergy Rhinol. 2018;8(6):713-728. doi: 10.1002/ alr.22094.

30. Rosenberg T, Fialla AD, Kjeldsen J, Kjeldsen AD. Does severe bleeding in HHT patients respond to intravenous bevacizumab? Review of the literature and case series. Rhinology. 2019;57(4):242-251. doi: 10.4193/Rhin18.289.

31. Abiri A, Goshtasbi K, Maducdoc M, Sahyouni 
R, Wang MB, Kuan EC. Laser-Assisted Control of Epistaxis in Hereditary Hemorrhagic

Telangiectasia: A Systematic Review. Lasers Surg Med. 2020;52(4):293-300. doi: 10.1002/lsm.23147.

32. Sena Esteves S, Cardoso C, Silva A, Abrunhosa J, Almeida E Sousa C. Nasal closure for the treatment of epistaxis secondary to hereditary hemorrhagic telangiectasia. Acta Otorrinolaringol Esp. 2016;67(6):345-348. doi: 10.1016/j.otorri.2015.12.002.

33. Buchberger AMS, Baumann A, Johnson F, et al. The role of oral anticoagulants in epistaxis. Eur Arch Otorhinolaryngol. 2018;275(8):2035-2043. doi: 10.1007/s00405-018-5043-z.

34. Rainsbury JW, Molony NC. Clopidogrel versus low-dose aspirin as risk factors for epistaxis. Clin Otolaryngol. 2009;34(3):232-235. doi: 10.1111/j.17494486.2009.01926.x.

35. Gomes P, Salvador P, Lombo C, Caselhos S, Fonseca R. Role of age and anticoagulants in recurrent idiopathic epistaxis. Acta Otorrinolaringol Esp. 2020;71(3):160-165. doi: 10.1016/j.otorri.2019.05.003.

36. Escabasse V, Bequignon E, Vérillaud B, et al. Guidelines of the French Society of Otorhinolaryngology (SFORL). Managing epistaxis under coagulation disorder due to antithrombotic therapy. Eur Ann Otorhinolaryngol Head Neck Dis. 2017;134(3):195-199. doi: 10.1016/j. anorl.2016.10.001.

37. Iqbal IZ, Jones GH, Dawe N, et al. Intranasal packs and haemostatic agents for the management of adult epistaxis: systematic review. J Laryngol Otol. 2017;131(12):1065-1092. doi: 10.1017/ S0022215117002055.

38. Mathiasen RA, Cruz RM. Prospective, randomized, controlled clinical trial of a novel matrix hemostatic sealant in patients with acute anterior epistaxis. Laryngoscope. 2005;115(5):899-902. doi: 10.1097/01. MLG.0000160528.50017.3C.

39. Murray S, Mendez A, Hopkins A, El-Hakim H, Jeffery CC, Côté DWJ. Management of Persistent Epistaxis Using Floseal Hemostatic Matrix vs. traditional nasal packing: a prospective randomized control trial. J Otolaryngol Head Neck Surg. 2018;47(1):3. doi: 10.1186/s40463-017-0248-5.

40. Lau AS, Upile NS, Lazarova L, Swift AC. Evaluating the use of Floseal haemostatic matrix in the treatment of epistaxis: a prospective, control-matched longitudinal study. Eur Arch Otorhinolaryngol. 2016;273(9):2579-2584. doi: 10.1007/s00405-016- 3948-y.

41. Le A, Thavorn K, Lasso A, Kilty SJ. Economic evaluation of floseal compared to nasal packing for the management of anterior epistaxis. Laryngoscope. 2018;128(8):1778-1782. doi: 10.1002/lary.27081.

42. Kunz SM, Holzmann D, Waser Y, Meerwein C, Deggeller M, Soyka MB. Does Length Matter? A Comparison of Rapid Rhino ${ }^{\mathrm{TM}}$ Nasal Packings for the Treatment of Epistaxis. Am J Rhinol Allergy. 2019;33(6):723-729. doi: 10.1177/1945892419864799.

43. Badran K, Malik TH, Belloso A, et al. Randomized controlled trial comparing Merocel and RapidRhino packing in the management of anterior epistaxis. Clin Otolaryngol. 2005;30: 333-337.

44. Knopfholz J, Lima-Junior E, Précoma-Neto D, Faria-Neto JR. Association between epistaxis and hypertension: a one year follow-up after an index episode of nose bleeding in hypertensive patients. Int J Cardiol. 2009;134(3):e107-e109. doi: 10.1016/j. ijcard.2008.01.018.

45. Varma A, Agrahari AK, Singh CV. Clinical Profile of Patients Presenting with Epistaxis and Hypertension: A Retrospective Clinical Study. Indian Medical Gazette. 2015; 320-323.

46. Min HJ, Kang H, Choi GJ, Kim KS. Association between Hypertension and Epistaxis: Systematic Review and Meta-analysis. Otolaryngol Head Neck Surg. 2017;157(6):921-927. doi: 10.1177/0194599817721445.

47. Kikidis D, Tsioufis K, Papanikolaou V, Zerva K, Hantzakos A. Is epistaxis associated with arterial hypertension? A systematic review of the literature. Eur Arch Otorhinolaryngol. 2014;271(2):237-243. doi: 10.1007/s00405-013-2450-z.

48. Payne SC, Feldstein D, Anne S, Tunkel DE. Hypertension and Epistaxis: Why Is There Limited Guidance in the Nosebleed Clinical Practice Guidelines? Otolaryngol Head Neck Surg. 2020;162(1):33-34. doi: 10.1177/0194599819889972.

49. Côrte FC, Orfao T, Dias CC, Moura CP, Santos M. Risk factors for the occurrence of epistaxis: Prospective study. Auris Nasus Larynx. 2018;45(3):471-475. doi: 10.1016/j.anl.2017.07.021.

50. Elwany S, Ibrahim AA, Soliman AI, Bazak R, Ibrahim HA. The significance of atherosclerosis in hypertensive patients with epistaxis. J Laryngol Otol. 2018;132(4):323-326. doi: 10.1017/ S0022215118000221. 